stellen der aufzählung der engel die gesamtsumme voraus: zwölf, vierzehn, zehn, sechs u.s.w., nur der nd. segen zeigt hier eine entstellung. Diese angabe der summe wird auch für unseren segen zu erwarten sein, deshalb ist min in das graphisch nahestehende niun zu ändern. Damit fällt dann auch die notwendigkeit, mit MSD. das reimwort geyn in ge zu ändern. Ob Schönbach falsch gelesen, oder die hs. falsch geschrieben hat, kann ich nicht mit sicherheit entscheiden, da die hs. nicht erreichbar ist. Wahrscheinlicher ist mir ein fehler in der hs. selbst, deren schreiber den segen offenbar nicht aus dem gedächtnis, sondern nach einer vorlage geschrieben hat. Der schreiber war m. e. ein Bayer, seine vorlage dagegen mitteldeutsch. Beim ersten wort heute gab er seiner heimischen mundart nach, später ist er bemüht, die md. sprachform der vorlage getreu wiederzugeben und schreibt formen wie engil, wedir, brengyn, worde, cruze, snide. $\mathrm{DaB}$ er, der doch wohl neun sprach, ein niun der vorlage leicht als min mißverstehen konnte, liegt auf der hand. Nicht denkbar wäre der umgekehrte vorgang, daß etwa ein mitteldeutscher schreiber eine bairische vorlage abschrieb; denn wenn eine solche vorlage heute schrieb, muß für sie auch eine form neun angenommen werden, die von einem mitteldeutschen, wenn er sie natürlich auch nicht selbst sprach, doch kaum als min mißverstanden oder verlesen werden konnte.

GIESSEN, 1. märz 1915.

KARL HELM.

\title{
ZUM CODEX PALATINUS 343.
}

Den zuerst von Görres 1817 in seinen 'Altdeutschen volksund meisterliedern' in seiner weise ausgebenteten codex palatinus 343, vielleicht die wichtigste der größeren älteren liederhandschriften des 16. jh.'s, hat uns erst Kopp in vollstiindigem abdruck zugänglich gemacht (Berlin 1905, leutsche texte des mittelalters 5). An seine an aufschliissen ïber die geschichte 
der betreffenden lieder wic an einzelbemerkungen zu den texten reiche ausgabe kniipfen die folgenden erörterungen einzelner stellen an.

3, 21 Sathan thuct dich anwehen, mocht er dich sturzen umb (: schueigen...ffrum). Weder der zweite teil der Bergreihen, aus dem Kopp die abweichenden lesarten anführt, noch die von Goedeke und Tittmann, Liederb. aus d. 16. jh. ${ }^{2}$ s. 246 genimnten älteren quellen geben eine variante. Der reim führt ohne weiteres auf das richtige anweigen, das, einmal von einem drucker mitteldeutscher herkunft mißverstanden, in verfälschter for'm anvehen in alle texte einging. Mhd. belege für anweigen im sinne von 'angreifen, anfechten, bedrängen', der auch hier gefordert wird, geben das Mhd. wörterb. 3, 556a und Lexer 3,742 aus denkmälern des 14. jh.'s, denen sich nun dieser jüngere beleg aus dem 16. als einziger nhd. gesellt. Kopp hätte erwähnen sollen, daß als verfasser dieser geistlichen contrafaktur Kunz Leffel zu gelten hat (vgl. Goedeke, Grundr. ${ }^{2}$ 2, 293).

In dem wunderschönen, von warmem und tiefem naturgefühl durchströmten frühlingsliede 32 singt der dichter (6): Es hatt die heit ir winderlileit gezogen ab, ir reiche hab hatt sich darein massieret; das darein geht sichtlich auf die unmittelbar vorhergehenden worte (4): des angers weyt stett lustigkleih bezieret. Aber was bedeutet massieret? Grimms wörterbuch kennt ein solches wort nicht, Kopp bucht es im wortverzeichnis (s.229), wie er meist tut, ohne den versuch einer deutung. Es ist sicherlich aus dem mhd. muosieren verderbt, das aus der ursprïnglichen bedeutung 'musivische, ausgelegte arbeit anbiingen' in die allgemeinere 'mustern, bunt färben' überging und demgemäß von erzeugnissen der kunst auf erscheinungen der natur übertragen werden konnte. Mhd. belege geben das Mlıd. wörterb. 2, 1,241 a und Lexer 1,2241, nhd. Grimms wörterb. 6,2739. Unser dichter gebraucht es wie ähnlich Jörg Schiller in der ersten strophe seines meisterlieds 'Des maien zeit' von 1505 (Zwickauer faksimiledrucke 25, von der satten färbung einer wiese im blumenschmuck wie einer der Bergreihen $(40,18)$ von dem zarten näslein der geliebten. Die an der letztgenannten stelle gebrauchte form mosirt gibt uns wohl auch an, welchen vocal wir in der form an unsrer stelle für das falsche $a$ einzusetzen haben. 
53, 1 Vor zeiten was ich lieb und werdt, die ich mir hett auserkoren: jetzundt hatt es sich ghar verkhert, es ist alles an ir verloren. Obwohl zwei parallele überlieferungen (Ambr. liederb. 28, 1; Euph. 9,41) abgesehen von der form war die lesung der anfangszeile bestätigen, so kann doch kein zweifel bestehen, daß was sich mit jenem dem älteren volksliedstil so geläufigen reflexivum beim verbum substantivum $\mathrm{zu}$ lesen ist, über das Grimm, Gramm. 4, 36 gehandelt hat. Der satzphonetische zusammenstoß der beiden $s$ bewirkte den verlust des einen, das er nicht mehr verstand, im sprachgefühl des ersten schreibers, während der zweite an einer andern stelle $(146,5)$ das auch ihm ungeläufige sprach sich durch sprach sie ersetzt hat, ohne sich an das dadurch entstandene doppelsubject sie das freurvlein $\mathrm{zu}$ stoßen.

55, 18 Frau Venus edle mein (: erkhenn); wörtlich dasselbe zeigt die überlieferung im Ambr. liederb. 253, 18. Der reim leitet auf die sichere besserung minn als die ursprüngliche lesart.

66, 24 Hoffart zoch Lucifer zu der helle, darnach wardt könig Meinratt sein geselle, der also mechtig wass und ungezerne, der sich gleich gott seim schopfer mass. Daß mit diesem hier neben Kosdras und Heraclius genannten könig Meinratt niemand anders als Nimrod gemeint ist, von dem die genesis $(10,8)$ berichtet: 'ipse coepit esse potens in terra et erat robustus venator coram domino', hätte Kopp im namenverzeichnis (s. 233), da er sonst verderbte namensformen erklärt, bemerken sollen.

90,4 recht wie das gruene gras geprosen aus eim anger weiss mit manchen bluemlen lilar; Kopp ändert geprosın in gesprosen. Obwohl auch eine parallele ïberlieferung (Ambr. liederb. 57,5) entsprossen bietet, möchte ich doch wenigstens auf die möglichkeit hinweisen, daß in geprosen etwas richtiges, altertümliches erhalten sein könnte. $\mathrm{Zu}$ den aus der nachklassischen zeit stammenden belegen für briezcn 'knospen treiben' im Mhd. wörterb. 1,260b, neben dem die ahleilung brozzen steht (ebenda 1, $261 \mathrm{a}$; Grimms wörterb. 2, 3!9!). k(mmmt. noch einer aus dem mönch von Salzburg $(36,12)$. \%wingend ist die beibehaltung der ïberlieferten lesart jedoch nicht.

In dem gedicht 106 werden eine ganze reilue von opforn der liebesleidenschaft aufgezïhlt, darmorer auch solche ans der antiken sage. Kowischen (irce und Herkules anf der cinch, 
Samson und Semiramis auf der andern seite finden sich hier die verse (140): Adam der lam durchs weib in not, den todt - hörest - cr auch empfieng. Natürlich gehört Adam zu den typischen beispielen der durch das schöne geschlecht betörten und ins ungliick gebrachten männlichkeit, aber daß auch sein tod mit diesem motiv in verbindung gebracht wird, hat in der genesis, der einzigen quelle, keinen anhalt. Daß in diesem satze ein verderbter eigenname steckt, zeigt die parallele überlieferung Arnt von Aichs, bei dem er so lautet: den todt IIorrestes auch enipfieng. Nun starb zwar Orestes nach der landläufigen sage an einem schlangenbiß, hatte aber infolge von liebesabenteuern nach seiner entsühnung vor dem areopag verfolgungen und gefahren genug auszustehen, daß seine aufnahme in die opferliste Amors gerechtfertigt erscheint. Auch in einem liede in Forsters sammlung $(1,124,2)$ erscheint er in einer solchen.

128, 12 do die taygen pirn stan. Kopp im wortverzeichnis erklärt (s. 230): 'pirn = birnen oder beeren?' Richtig ist natürlich nur die erste erklärung, wie die parallelstellen in Grimms wörterb. 11, 236 deutlich zeigen, zumal auch von teigen beeren zu reden nirgends sprachüblich sein dürfte, wohl aber von teigem obst.

145, 55 Cato thuet uns beschreiben mit seiner scharpffen lisst: ich sollt mein laid vermengen mit freud zue diser frist. Gemeint ist disticha Catonis 3,7: 'Interpone tuis interdum gaudia curis, ut possis animo quemvis sufferre laborem.' Von den in Zarnckes ausgabe gedruckten deutschen fassungen klingt keine näher an unsre stelle an, wohl aber ein zitat der gleichen stelle in einem liede in Forsters sammlung $(3,48,3)$.

Für die sicherlich in 152, 35 in erlan sitt steckende verderbnis ist mir keine heilung gelungen, weshalb ich ausdrücklich noch einmal den finger auf diese wunde legen möchte, da mich Kopps erklärungsversuch 'nach art einer schwankenden (bisweilen sinnbildlich und sprichwörtlich angeführten) 'erle' in keiner weise befriedigt.

Ebenso bedürfen die literarischen anspielungen in dem gedicht 154 noch an zwei stellen der aufhellung. Der in der höfischen erzählungsliteratur sehr gut beschlagene verfasser, dem die bezeichnung dichter allerdings $\mid$ zuw viel $\cup$ ehre antun 
würde, nennt in jeder der sieben strophen, aus denen das gedicht ursprünglich nur bestand, wie die von Kopp verglichene parallele ïberlieferung zeigt, als trost seiner leiden einen erlauchten leidensgefährten aus den gefilden der epischen literatur. Fünf davon sind bekannt: Parzival, als ihm das erste mal der gral verschwand; Tristram, als ihm das schwarze segel angekündigt wurde; Friedrich von Schwaben, als er die drei tauben erlegte (vgl. auch 145,73); der ritter mit dem bocke, als er der gnade seiner dame verlustig ging (Konrad von Stoffels Gauriel von Muntabel; vgl. Kopp im namenverzeichnis s. 233); Hektor, der vor Troja bleiben mußte. Von den beiden übrigen dürfte Flordamur der ward in freud zerspallten (24) einer verlorenen dichtung angehören, wenigstens weisen die erhaltenen dichtungen der höfischen epik keinen helden dieses namens auf; an der richtigen überlieferung des namens zu zweifeln liegt zunächst kein grund vor. Endlich Artus der hoch leut (= leit) ungemach, da im erschyn ein frembdes höre uff weitem feld, darab er het verdriesscn, nach dem er sant, gros freud er fandt (27): hier scheint eine situation aus des Strickers Daniel von dem blühenden tal vorzuschweben, die aus Bartschs inhaltsauszug (Karl der große s. XVIII) ersichtlich ist.

196, 41 wann ich nit bin eylengeschlecht. Kopp hat im wortverzeichnis (s. 228) dies adjectiv, die directe fortsetzung des mhd. iuwelenslaht (Wolfr. lied. 5, 20; Freid. 145, 19), nicht aufgenommen. Da es auch in Grimms wörterbuch fehlt (nur die beiden mhd. belege sind dort gegeben), so sei hier auf noch einen frühnhd. beleg hingewiesen: maynst $d u$, wir seyen euwlengeschlechtt? (Albr. v. Eyb 2, 92, 16).

JENA, 31. januar 1915.

ALBERT LEITZMANN. 